\title{
Recommendations Based on Purchase Patterns
}

\author{
Haiyun $\mathrm{Lu}$
}

\begin{abstract}
We propose an approach of recommendation based on purchase patterns. The purchase history of users is analyzed to find their purchase patterns related to user behavior. These patterns are then used to predict the category of next possible purchase in a particular location. The proposed approach is experimented on real transaction data. Synthetic and simulation tests are conducted to evaluate the performance. Results show that it performs better than the baseline sequential pattern analysis.
\end{abstract}

Index Terms-Recommendation systems, purchase patterns, sequential pattern analysis.

\section{INTRODUCTION}

With the rapid development of computer technologies and the Internet of Things, e-commerce industry continues to boost its business. Personalized recommendation systems are of great interest for the e-commerce industry. Such systems are used, for ex-ample, to recommend products that may interest a particular buyer. Recommendation systems allow buyers to find what they need without browsing through catalogs. They also enable sellers to increase the conversion rate by attracting buyers with those products they might prefer Thus, benefits for both parties are furnished by recommendation systems. As a result of this importance, many work and study has been devoted to the knowledge and techniques for recommendation systems. Major approaches include content-based filtering [1]-[4], collaborative filtering [5]-[10], rule-based [11]-[13], and hybrid approaches [14]-[18].

As mobile devices are gaining massive popularity, mobile e-commerce becomes much more important to the industry. Consumers could use the mobile device to complete a transaction anywhere and anytime. In the context of mobile environment, a lot more information about buyers can be obtained, such as location. Location based recommendation, in this case, is preferred for business such as restaurants [19] and tourist attractions [20], and is often related to social networks [21].

When investigating recommendation mechanisms of the mobile version of an online shopping website for coupon deals, it is found that, in real business case, the information about customers is limited. In most cases, information about customer profile, product preference or product ratings, is not available. The most relevant information about a particular customer is her purchase history and current location. A straightforward approach is to recommend deals that are in the vicinity. Such recommendation will suffer from a higher

Manuscript received April 10, 2014; revised June 25, 2014.

Haiyun Lu is with SAP Research \& Innovation, Singapore (e-mail: hai.yun.lu@sap.com). probability of inaccuracy.

With regard to this problem, we propose a novel approach to analyze the purchase history of customers to find their purchase patterns, and apply them to location based recommendations. The objective is to achieve higher accuracy for the recommendation system.

\section{RELATED WORK}

There are generally four types of recommendation systems: content-based filtering, collaborative filtering, rule-based and hybrid approaches.

Content-based filtering system performs recommendation in steps. First, an item profile consisting of a set of features is extracted for each item. Second, user profiles are generated based on features of items that are purchased by each user. Then similarity scores between user profiles and item profiles are calculated to finally recommend items with top similarity scores. This technique was used to recommend documents such as news [1], web pages [2], movies [3], and books [4]. The features used in building profiles are often a set of keywords. The limitations of these systems are that recommendations are always similar to items that a user has already purchased, and it is difficult to recommend items for new users.

Collaborative filtering systems are built on the assumption that a good way to find interesting content is to find other people who have similar interests and then recommend items rated highly by those similar users [6]. There are two general classes of collaborative filtering algorithms. Memory based algorithms operate over the whole user database to make recommendations [5], [7]. Model based collaborative filtering, in contrast, learns a model from the user database, which is then used for recommendations [8]-[10]. Collaborative filtering recommendation systems, however, also have limitations that it is difficult to recommend items for new users, to recommend items which have not been rated before, and to recommend when rating information is insufficient.

Rule-based approach is a simple but popular way of recommendations. Rules are usually derived from database of previous transactions. Aggarwal et al. [11] discovered localized association rules among items that are purchased together, which are helpful for target marketing. Cho et al. [12] used data mining techniques such as web usage mining, decision tree induction, association rule mining to build a rule-based recommendation system. Huang \& Huang [13] proposed a sequential pattern based system that predicts customer's time-invariant purchase behavior for food items in a super market.

Hybrid approach combines multiple techniques to overcome the limitations of individual systems [14]-[18]. For 
example, a linear combination of rule-based and collaborative filtering recommendations considers customers' purchase sequences over time as well as their purchase data for latest period [17].

When applying these recommendation techniques to mobile environment, we are facing some new challenges as well as new opportunities. The mobile screens are smaller and thus few products are browsed. As a result, it is difficult to use users' browsing behavior in methods like collaborative filtering. However, more information can be obtained from users' mobile devices, such as location, dwell time and direction.

Location has been widely used in mobile environment to provide value-add services. For recommendation systems related to location based business such as restaurants or tourist attractions, the information of location can be helpful. For example, Park et al. [19] aimed at recommending restaurants. Their system use a Bayesian network that models the probabilistic influences of the user's personal profile and contextual information on the restaurant attribute values. Bao et al. [21] used location history of a user to derive personal preferences and social opinions mined from local experts to facilitate personalized location recommendations.

In this paper, we are targeting at a problem with limited amount of user information. User profiles, preferences, location histories, item ratings are not available. Furthermore, in the mobile shopping website for coupon deals, the life span of deals is only several days. All these limitations and constraints make it difficult to apply existing techniques to build recommendation systems. Therefore, we are proposing a novel approach to analyze the purchase history of the user, find their purchase patterns related to the user behavior and then predict information of the next possible purchase in a particular location.

\section{PURChase PATtern ANALYsis}

Purchase history of a user is a series of transactions ordered by the transaction time. Due to the nature of coupon deals, information such as deal titles and descriptions are not following any standard, and thus make it hard to use. In this paper, we are solely looking at the category information of the transaction. Thus the purchase history in this context is just the category sequence. For example, a user has 4 transactions with category sequence "BAAC". It means that the first purchase is a deal in category $\mathrm{B}$, the second and the third one are both in category $\mathrm{A}$ and the last one is in category $\mathrm{C}$.

Given a set of category sequences, the problem is to find purchase patterns related to the user behavior that can be used to make recommendations. Note that in this case, the recommendation will be the category as well.

A classical approach to solve this type of problem is to use sequential pattern analysis (SPA). In this approach, frequently occurring patterns are extracted from all subsequences of the given set of sequences. Each pattern is associated with a support value. Then all subsequences of a target user's sequence data is enumerated to match with the first part of identified patterns. The other part of a matching pattern becomes a candidate of the recommendation. The support values are summed up for each associated candidate, and the top scored candidate will be recommended.

However, this approach is not the most suitable for our problem. In real business, purchase histories of users vary from 1 transaction to up to 100 transactions, and the number of users is generally very large. Using sequential pattern analysis, the patterns derived are often associated with a very small support value, such as 0.01 . Also, this approach uses all subsequences, which may derive patterns relating distant transactions. Such patterns may not be relevant because coupon deals have short life spans and recency is important.

Therefore, we propose a novel approach to analyze purchase patterns by using consecutive subsequences. Consecutive subsequences are a subset of all subsequences. The consecutiveness ensures the recency of extracted patterns. For example, a user's purchase history is "BBAACCB", and then the consecutive subsequences are:

- Length 1: B, A, C

- Length 2: BB, BA, AA, AC, CC, CB

- Length 3: BBA, BAA, AAC, ACC, CCB

- Length 4: BBAA, BAAC, AACC, ACCB

- Length 5: BBAAC, BAACC, AACCB

- Length 6: BBAACC, BAACCB

- Length 7: BBAACCB

Among these subsequences, we focus on those with length smaller or equal to 6 . This is to avoid long patterns, as we aim to maintain recency.

An additional step is applied to further generalize the consecutive subsequences. Category information is replaced by the order of appearance, such that the purchase patterns extracted can better represent user behaviors. For example, above mentioned consecutive subsequences are further generalized as:

- Length 1: 1

- Length 2: 11, 12

- Length 3: 112, 122

- Length 4: 1122, 1223

- Length 5: 11223, 12233

- Length 6: 112233, 122331

Note that subsequences "BB", "AA" and "CC" are now generalized as the same " 11 ". Subsequences "BA", "AC" and "CB" are now generalized as " 12 ".

Next, all eligible subsequences from users are analyzed to build a conditional probability model. This model computes the conditional probability of a category matching the next purchase based on a given sequence. The conditional probability is given by,

$$
P(N=c \mid H=s)=\frac{P(H=s \cap N=c)}{P(H=s)}
$$

where $N=c$ denotes the event of a category $c$ matching the next purchase and $H=s$ denotes the event of category sequence $s$ being the purchase history. For example, if there are $x$ occurrences of pattern " 1 " and $y$ occurrences of pattern " 12 ", then probability of " 2 " matching next purchase given a purchase history " 1 " is given by, $P(N=$ " 2 " $\mid H=$ " 1 " $)=y / x$.

With the conditional probability model, we are able to make recommendations. For each possible category, the probability of such category being the next purchase is 
computed based on the given purchase history. Here, eligible suffix subsequences of the target user's purchase history are used. For example, if a user with purchase history "BBAACCB" is the target user, the following suffix subsequences need to be examined: "B", "CB", "CCB", "ACCB" and "AACCB". These suffix subsequences are served as conditions to compute the conditional probability. Suppose we are going to compute the probability of category "B" matching the next purchase, then $N=$ " $B$ " and there are five different cases of $H$ in (1). Note that the generalized form of subsequences is used in the computation. Five probabilities are computed as follows and they are summed up to be the score of category "B" for recommendation.

- $P(N=$ " $B$ " $\mid H=$ " $B$ " $)=P(N=$ " $1 " \mid H=$ " 1 ")

- $P(N=$ " $B$ " $\mid H=$ " $C B$ " $)=P(N=$ " 2 " $\mid H=$ " 12 " $)$

- $P(N=$ " $B$ " $\mid H=$ " $C C B$ " $)=P(N=$ " 2 " $\mid H=$ " 112 ")

- $P(N=$ " $B$ " $\mid H=$ " $A C C B ")=P(N=$ "3" $\mid H=$ " 1223 " $)$

- $P(N=$ " $B " \mid H=$ " $A A C C B ")=P(N=$ " 3 " $\mid H=$ ="11223" $)$

After that all possible candidates of $N$ are examined, the one with highest score is selected as the final recommendation. This recommendation is experimented on mobile devices for location based recommendation.

\section{EXPERIMENTS}

At an arbitrary location, coupon deals in the vicinity are to be recommended to the user. Since the nature of mobile usage, only limited number of deals shall be recommended. Thus it is important to select deals that are more likely to be purchased by the user. Our proposed method extracts purchase patterns from purchase history to model the user behavior and recommend the category of the next possible purchase.

\section{A. Data Set}

The data set used in our experiments is real transactions in an online coupon deal website. There are 27797 users who have at least 2 transactions in the purchase history within a time period of four months. There are 2072 different deals categorized in 5 categories.

During the experiment, the data set is randomly divided into two halves, one as training data set and the other as testing data set. The training data set is used to extract purchase patterns and build conditional probability model. After training, 273 different patterns are extracted. The test data set is then used to test the performance of recommendation.

\section{B. Experimental Settings}

For a particular user in the test data set, the user's transaction history is divided into two parts. The first part is considered as purchase history and it is used by the conditional probability model to make recommendations. The second part is the next purchase after first part, and it is considered as recommendation ground truth. A strict setting is used in the experiment, such that the second part only contains 1 transaction.

Assume that a user is located near a set of available coupon deals. The experiment aims to predict the correct category of the user's next purchase, so as to recommend the deals that are the most likely to be accepted.

\section{Synthetic Test}

To test the performance of recommendation, we first conduct a synthetic test. Assume a set of available coupon deals covers $n$ categories $(2 \leq n \leq 5)$. All possible sets, which include the ground truth category, are tested for all users in the test data set.

For the purpose of comparison, we also conducted experiment using sequential pattern analysis (SPA). The minimum support value is set to 0.01 and 101 different patterns are extracted.

As the ground truth is of size 1 for each user case, the standard measurement precision and recall are actually the same. Precision measurements of both experiments are illustrated in Fig. 1. Our approach achieves higher precision in all tests.

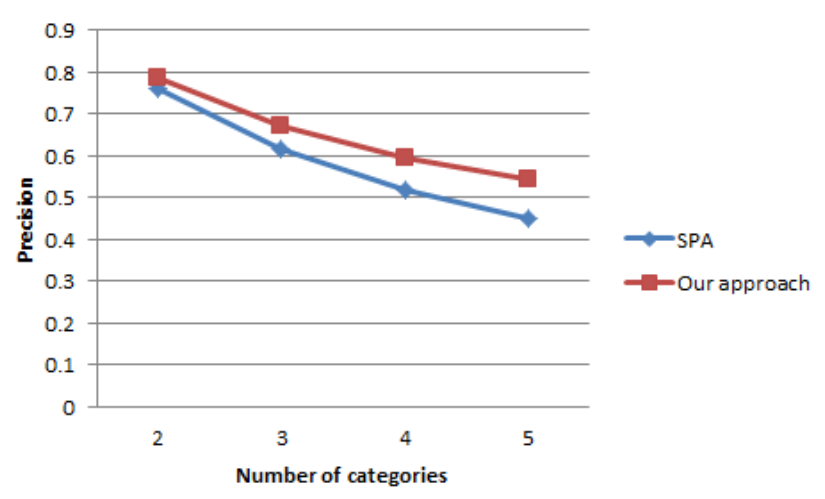

Fig. 1. Precision of recommendations by SPA and our approach.

\section{Simulation Test}

Since our method is targeting at recommendations for mobile users, we simulated the location of users to run the tests against real deals around the location. 10000 different locations in the city are uniformly generated and each location is in the vicinity of deals from at least two categories. Each user in the test data set is put to the location and recommendations are made according the same model trained in previous experiment.

If we consider only cases when ground truth is included in the set of available deals around a location, the precision of recommendations is 0.662 . This matches with the expectation from synthetic tests. If we consider all the cases, the precision is dropped to 0.409 . This is because in many cases, the recommendation cannot be successful as the deals around the location are limited.

The overall experiments are very successful. It shows that our proposed approach is able to select the correct category to recommend based on purchase patterns.

\section{CONCLUSION}

In this paper, we presented an approach of making recommendation based on purchase patterns. The purchase history of users is analyzed to find their purchase patterns related to user behavior. These patterns are then used to predict the category of the next possible purchase in a 
particular location. We conducted synthetic and simulation tests to evaluation the performance of our approach. Compared to a typical SPA approach, our approach achieved higher precision. However, there is still room for further research. For example, keywords and images of coupon deals can be incorporated into the recommendation system to overcome the limitation posed by locations and to enhance the flexibility of recommendation.

\section{REFERENCES}

[1] K. N. Lang, "Learning to filter netnews," in Proc. the 12th International Machine Learning Conference, 1995.

[2] M. Pazzani and D. Billsus, "Learning and revising user profile: the identification of interesting web sites," Machine Learning, vol. 27, no. 3, pp. 313-331, 1997.

[3] C. Basu, H. Hirsh, and W. Cohen, "Recommendation as classification: Using social and content-based information in recommendation," in Proc. 15th National Conference on Artificial Intelligence, 1998.

[4] R. J. Mooney and L. Roy, "Content-based book recommending using learning for text categorization," in Proc. ACM SIGIR '99 Workshop on Recommender Systems: Algorithms and Evaluation, Berkeley, CA, 1999.

[5] P. Resnick, N. Iacovou, M. Suchak, P. Bergstrom, and J. Riedl, "Grouplens: An Open Architecture for Collaborative Filtering of Netnews," in Proc. the ACM 1994 Conference on Computer Supported Cooperative Work, 1994.

[6] J. S. Breese, D. Heckerman, and C. Kadie, "Empirical Analysis of Predictive Algorithm for Collaborative Filtering," in Proc. the 14th Conference on Uncertainty in Artificial Intelligence, 1998.

[7] J. Delgado and N. Ishii, "Memory-based weighted-majority prediction for recommender systems," presented at ACM SIGIR '99 Workshop on Recommender Systems: Algorithms and Evaluation, 1999.

[8] T. Hofmann and J. Puzicha, "Latent Class Models for Collaborative Filtering," in Proc. International Joint Conference on Artificial Intelligence, 1999.

[9] D. M. Pennock, E. Horvitz, S. Lawrence, and C. L. Giles, "Collaborative Filtering by Personality Diagnosis: A Hybrid Memoryand Model-Based Approach," in Proc. the 16th Conference on Uncertainty in Artificial Intelligence, 2000.

[10] L. Si and R. Jin, "Flexible mixture model for collaborative filtering," in Proc. 20th International Machine Learning Conference, 2003.

[11] C. C. Aggarwal, C. Procopiuc, and P. S. Yu, "Finding localized associations in market basket data," IEEE Transactions on Knowledge and Data Engineering, vol. 14, no. 1, pp. 51-62, 2002.

[12] Y. H. Cho, J. K. Kim, and S. H. Kim, "A personalized recommender system based on web usage mining and decision tree induction," Expert systems with Applications, vol. 23, no. 3, pp. 329-342, 2002.
[13] C. L. Huang and W. L. Huang, "Handling sequential pattern decay: developing a two-stage collaborative recommendation system," Electronic Commerce Research and Applications, vol. 8, no. 3, pp. 117-129, 2009.

[14] Y. B. Cho, Y. H. Cho, and S. H. Kim, "Mining changes in customer buying behavior for collaborative recommendations," Expert System with Applications, vol. 28, no. 2, pp. 359-369, 2005.

[15] Y. Y. Shih and D. R. Liu, "Product recommendation approaches: collaborative filtering via customer lifetime value and customer demands," Expert Systems with Applications, vol. 35, no. 1-2, pp. 350-360, 2008.

[16] Y. Jiang, J. Shang, and Y. Liu, "Maximizing customer satisfaction through an online recommendation system: a novel associative classification model," Decision Support Systems, vol. 48, no. 3, pp. 470-479, 2009.

[17] D. R. Liu, C. H. Lai, and W. J. Lee, "A hybrid of sequential rules and collaborative filtering for product recommendation," Information Sciences, vol. 179, no. 20, pp. 3505-3519, 2009.

[18] K. Choi, D. Yoo, G. Kim, and Y. Suh, "A hybrid online-product recommendation system: Combining implicit rating-based collaborative filtering and sequential pattern analysis," Electronic Commerce Research and Applications, vol. 11, no. 4, pp. 309-317, 2012.

[19] M. H. Park, J. H. Hong, and S. B. Cho, "Location-based recommendation system using Bayesian user's preference model in mobile devices," Ubiquitous Intelligence and Computing, vol. 4611, pp. 1130-1139, 2007.

[20] M. D. Dunlop, B. Elsey, and M. M. Masters, "Dynamic visualisation of ski data: a context aware mobile piste map," in ACM International Conference Proceeding Series, A. D. Cheok and L. Chittaro Ed., vol 309, pp. 375-378, 2007.

[21] J. Bao, Y. Zheng, and M. F. Mokbel, "Location-based and preference-aware recommendation using sparse geo-social networking data," in Proc. the 20th International Conference on Advances in Geographic Information Systems, pp. 199-208, 2012.

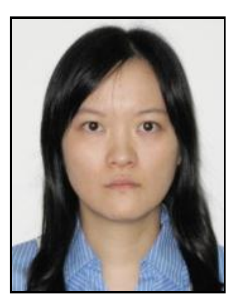

Haiyun $\mathrm{Lu}$ obtained $\mathrm{PhD}$. in computer science from National University of Singapore in 2012, and Bachelor of Computing from the same university.

She is currently a researcher with SAP Research \& Innovation in Singapore. Her research interests include computer vision and machine learning. 